OD $66<x<8$

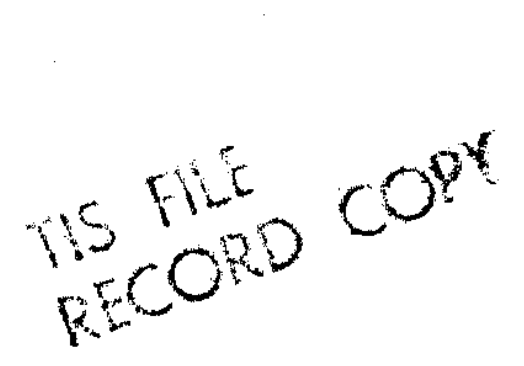

24

\title{
CALCULATIONS FOR ESTIMATING THE EQUILIBRIUM WASHOUT OF TRITIUM
}

P. E. LONG, JR.

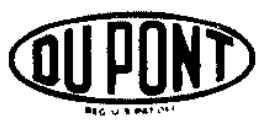

\section{E. I. DU PONT DE NEMOURS AND COMPANY SAVANNAH RIVER LABORATORY AIKEN, SOUTH CAROLINA 29801}




\section{NOTICE}

This report was prepared an account of work sponsored by the United States Government. Neither the United Stetes nor the United States Energy Research and Development Administration, nor any of their contractors, subcontractors, or their employees, makes any warranty. express or implied, or assumes any legal liability or responsibility for the accuracy, completeness or usefulness of any information, apparatus, product or process disclosed, or represents that its use would not infringe privatoly owned rights.

\begin{tabular}{|c|} 
Printed in the United States of America \\
Available from \\
National Technical Information Service \\
U.S. Department of Commerce \\
s285 Port Royal Road \\
Springfield, Virginia 22161 \\
Price: Printed Copy $\$ 4.50 ;$ Microfiche $\$ 3.00$
\end{tabular}


DP -1443

Distribution Category: UC-11

CALCULATIONS FOR ESTIMATING THE EQUILIBRIUM WASHOUT OF TRITIUM

by

P. E. Long, Jr.

Approved by

T. V. Crawford, Research Manager

Environmenta] Transport Division

Publication Date: January 1978

E. I. DU PONT DE NEMOURS AND COMPANY SAVANNAH RIVER LABORATORY AIKEN, SOUTH CAROLINA 29801

PREPARED FOR THE U.S. DEPARTMENT OF ENERGY UNDER CONTRACT AT(07.211 


\section{ABSTRACT}

The equilibrium washout of tritiated water vapor (HTO) and tritium gas (HT) downwind from an instantaneous point source can be estimated by simple formulas. Derivations for three different models (Fickian Puff, Leaky Puff, and Leaky Garbage Can) are given for estimating the washout $/ \mathrm{m}^{2}$ from a puff. Two models are given (Leaky Garbage Can and Fickian Puff) for calculating the fraction of pollution remaining in a puff after it has traveled over an arbitrary distance. The relationship between washout and dry deposition problems is also discussed in this report.

The Leaky Garbage Can model is similar to the Leaky Puff mode1, but HTO is assumed to be well mixed beneath an inversion lid which the HTO cannot penetrate.

The following are some key results from the investigation:

- The washout of HT is probably slight because it is almost in-. soluble in water.

- The washout of HTO is mathematically equivalent to (but physically different from) dry deposition, provided certain assumptions are satisfied.

- The washout "deposition velocity" of HTO can easily be much greater than conventional dry deposition velocities of gases or particulates.

- HTO can be washed out within several tens of kilometers under moderate rain. 


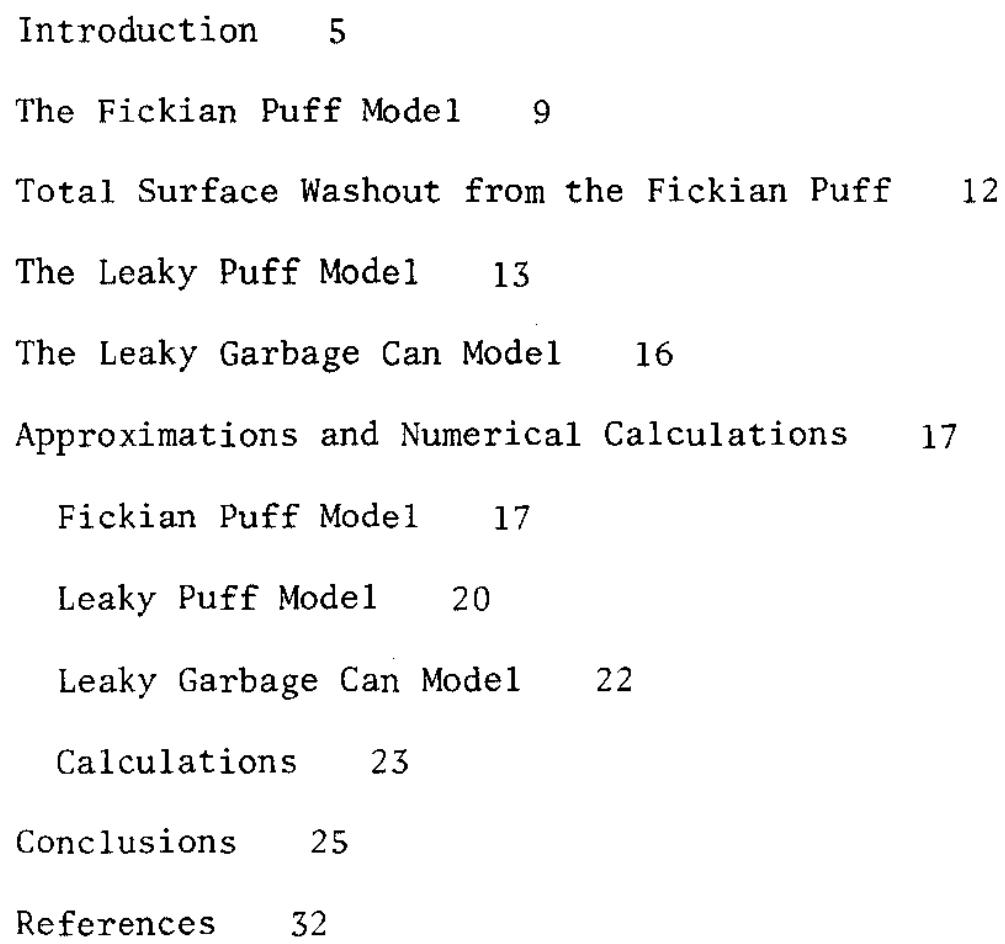




\section{LIST OF FIGURES}

1 HTO Remaining in Puff Calculated by Leaky Puff Model 27

2 Effect of Increased Rain Rate 28

3 Comparison of Calculations with Fickian Puff and Leaky Puff Models 29

4 HTO Remaining in Puff Calculated by the Leaky Garbage Can Model with Fixed and Rising Lids 30

5 Comparison of Calculations with Fickian Puff and Leaky Garbage Can Models 31 


\section{INTRODUCTION}

A number of papers on particulate scavenging have been collected by Engelmann and Slinn. ${ }^{1}$ The removal of particles, however, is different mathematically and physically from the scavenging process by which gases like HT and HTO are removed from puffs. The problem of pollution scavenging from within frontal and convective clouds (rainout) is not explained in this report; all the relations require the puff to be below the offending cloud (washout).

This paper discusses derivations for three different models (Fickian Puff, Leaky Puff, and Leaky Garbage Can) which are given for estimating the washout $/ \mathrm{m}^{2}$ from a puff. The Leaky Garbage Can and Fickian Puff models are used for calculating the fraction of pollution remaining in a puff after the puff has traveled an arbitrary distance. The relationship between washout and dry deposition problems is also discussed.

To estimate the equilibrium washout for each of the three mode1s, the following assumptions are made: a steady wind, a steady rain, and washout flux proportional to the concentration of the puff at ground level. The Fickian Puff model requires the vertical eddy transfer coefficient to be constant. The Leaky Puff model is assumed to be Gaussian, and the washout effect is simulated by an ever-diminishing effective source term.

An averaging process could be used in which a very patchy rain, like a summer thundershower, can be replaced by a steady rain rate computed by averaging over the variable rain. If only a small fraction of HTO were washed out during the heaviest portions of the rain, averaging may be justifiable. However, if a puff passes under a vigorous thunderstorm, the puff can be depleted quickly; the process is nonlinear, and averaging is not allowable. The chance of a puff from an accidental release passing under a vigorous cell is small.

Studies by Barr $^{2}$ show that an occluded midlatitude cyclone may be broken into four zones:

1. Steady stratiform rain ahead of a developing wave covering an area of about $10^{4} \mathrm{~km}^{2}$ is distributed within a banded structure. 
2. Patchy stratiform rain within a $10^{3}-\mathrm{km}^{2}$ area is near the storm center. Rain rates as high as $6 \mathrm{~mm} / \mathrm{hr}$ can be found within about $3:$ of the area.

3. Frontal convective showers within a $50-\mathrm{m}$ to $100-\mathrm{m}$ wide band cover less than $1 \%$ of the area and have rates exceeding $6 \mathrm{~mm} / \mathrm{hr}$.

4. Postfrontal convective showers cover a large area; only a small portion of the total area has much rain.

The concentration of HTO within a falling raindrop is in equilibrium with its surrounding atmosphere, except within a few decades of stack heights downwind from the source. ${ }^{3}$ The equilibrium occurs because the puff has spread sufficiently by the time it has traveled about $1 \mathrm{~km}$, which gives a falling raindrop ample time to approach equilibrium. A puff $1 \mathrm{~km}$ from its source has a four-standard deviation "diameter" of about $80 \mathrm{~m}$. Equilibrium case and calculations that account for the past history of the raindrop for downwind distances ranging from $100 \mathrm{~m}$ to $1.6 \mathrm{~km}$ are compared, and the equilibrium approximation is found to be poor for HTO at a distance of $100 \mathrm{~m}$ but good at a distance of $1.6 \mathrm{~km} .^{3}$ All relations in this report are derived from assumed equilibrium conditions. Therefore, the washout flux to the surface is proportional to the product of the surface concentration of the vapor and the rain rate. Thus,

$$
\text { Flux }=\alpha c(x, y, z=0, t) R
$$

where

$$
\begin{aligned}
& \alpha=\text { proportionality constant } \\
& c=\text { puff's concentration of HTO } \\
& \mathrm{R}=\text { rain rate }
\end{aligned}
$$

If the rain rate is measured in units of mass $\operatorname{area}^{-1}$. time $e^{-1}$, then $\alpha R$ has the dimensions of velocity. The product $\alpha R$, to be denoted by $V_{W}$, is analogous to deposition velocity in the dry deposition of pollution to the foliage and to the ground. The physical mechanisms are different for these two processes (dry deposition and washout).

The "constant" of proportionality (a function of temperature) can be evaluated by using Raoult's Law for the partial pressure of a solute dissolved in a solvent and Dalton's Law of Partial pressures. These laws for HTO are

$$
\mathrm{P}_{\text {HTO }}=X_{\text {HTO }}^{S} P_{O}^{S} \text { (Raou1t's Law) }
$$

and

$$
\mathrm{P}_{\text {HTO }}=\mathrm{x}_{\text {HTO }}^{\mathrm{g}} \mathrm{p}_{\mathrm{O}}^{\mathrm{g}} \text { (Dalton's Law) }
$$


where

$\mathrm{P}_{\text {HTO }}=$ partial pressure of HTO vapor

$\mathrm{x}_{\mathrm{HTO}}^{\mathrm{S}}$ and $\mathrm{x}_{\mathrm{HTO}}^{\mathrm{g}}=\begin{aligned} & \text { mole fractions of } \mathrm{HTO} \text { in a raindrop and } \\ & \text { surrounding air, respectively }\end{aligned}$

$\mathrm{P}_{0}^{\mathrm{S}}$ and $\mathrm{P}_{0}^{\mathrm{g}}=$ pressures above pure liquid HTO and the total pressure of the air vapor combination, respectively.

Equation 2, Equation 3, and the accurate approximation that HTO is dilute in all practical cases gives

$$
c_{\text {Sol }} \doteq \frac{\rho_{\mathrm{H}_{2} \mathrm{O} / \rho_{\text {air }}}}{\text { Mixing Ratio }} \mathrm{c}
$$

where

$\mathrm{c}_{\mathrm{SO} 1}=$ the concentration of HTO in a raindrop

$\rho_{\mathrm{H}_{2} \mathrm{O}}$ and $\rho_{\text {air }}=$ densities of water and air

Mixing Ratio = amount of water vapor for each unit mass of dry air in the mixture

In deriving Equation 4, the vapor pressures of pure $\mathrm{H}_{2} \mathrm{O}$ and HTO are assumed to be almost equal. Because it must be true that

$$
\text { Flux }=\frac{R}{\rho_{\mathrm{H}_{2} \mathrm{O}}} c_{\text {Sol }} \text {, }
$$

it follows that

$$
\text { Flux }=\frac{R c(x, y, z=0, t)}{\text { Absolute Humidity }} \equiv V_{w} c(x, y, z=0, t)
$$

Absolute humidity is the mass of $\mathrm{H}_{2} \mathrm{O}$ vapor for each volume of air.

At room temperature, $V_{w} \doteq 2 R_{m m}$. Here, $R_{m m}$ is the rain rate measured in $\mathrm{mm} / \mathrm{hr} ; V_{W}$ is the washout deposition velocity expressed in $\mathrm{cm} / \mathrm{sec}$. Because rain rates are frequently greater than $1 \mathrm{~mm} / \mathrm{hr}$, washout deposition velocities will usually be greater than commonly cited dry deposition velocities ( $1 \mathrm{~cm} / \mathrm{sec}$ ).

Dimensional analysis suggests that a puff released from the surface, confined to a layer of height $\mathrm{H}$, and moved downwind at a constant speed U should be depleted significantly over a distance 
$\mathrm{L}=\frac{\mathrm{UH}}{\mathrm{V}_{\mathrm{W}}}$. For $\mathrm{H}=500 \mathrm{~m}, \mathrm{U}=5 \mathrm{~m} / \mathrm{sec}$, and $\mathrm{R}_{\mathrm{mm}}=5 \mathrm{~mm} / \mathrm{hr}$, the depletion scale $\mathrm{L}=25 \mathrm{~km}$. The Leaky Garbage Can model (Equation 44) confirms the simple dimensional relation presented here.

The Fickian Puff and Leaky Puff models are fairly general and allow for elevated sources and power law variations of $\sigma$ with $x$. In the following derivations, the distance downstreăm from the source is denoted by $x$, and the standard deviation of the vertical spread of the puff is denoted by $\sigma_{z}$.

A power law form of $\sigma_{z}(x)$ based upon Högström's ${ }^{3}$ data was chosen for reasons of simplicity. Extending Högström's power law beyond several kilometers is questionable, yet this is done for all the numerical examples presented. Other expressions for $\sigma_{z}(x)$ which have different forms for large and smal1 $x$ could have been used but would have complicated the analysis.

The washout for $\mathrm{HT}$ and $\mathrm{T}_{2}$ will not be explicitly calculated in this report because of the relative insolubility of $H T$ and $T_{2}$. The derivation for the washout deposition velocity of $H T$ and $T_{2}$ is similar to that for HTO, but $P S$ in Raoult's Law (Equation 2) must be replaced by Henry's Law constant ${ }^{4}$ (the "constant" depends on temperature). Henry's Law constant is larger than $\mathrm{P}_{\mathrm{O}}^{\mathrm{S}}$ by a factor of about $10^{6}$ thereby making $V_{w}$ for $T_{2}$ and $H_{T} 10^{6}$ smaller than that for HTO. The models in the following sections are intended for the calculation of the washout of HTO.

Results for the Fickian Puff and Leaky Puff models were compared. These results agree fairly well with each other provided the puffs retain a reasonable amount of their original HTO. The discrepancies arise because the washout boundary value problem is directly solved by the Fickian Puff model while a mathematical subterfuge is used to account for the total amount of material remaining in the Leaky Puff. The Leaky Puff model keeps its Gaussian nature regardless of the intensity of the rain and the amount of material left in the puff. The Fickian Puff model allows the puff to change its shape as material is washed out. The profile of the concentration changes markedly as the Fickian Puff is depleted.

Finally, an admittedly heuristic argument extends the Fickian Puff to cases other than those governed by pure Fickian diffusion. The argument achieves its purpose in a formal sense.

The relations in this report for washout may be useful for dry deposition problems. 


\section{THE FICKIAN PUFF MODEL}

The washout from a puff can be solved in closed form if Fickian diffusion (constant eddy diffusion coefficients) is assumed. However, because the concentration of a puff is a product of separate solutions for each of three dimensions, vertical Fickian diffusion is sufficient. Initially the diffusion is completely Fickian, and later in the development of this model, the condition of Fickian diffusion in the $X-Y$ plane is relaxed. equation

The problem to be solved is a three-dimensional diffusion

$$
\frac{\partial c}{\partial t}+U \frac{\partial c}{\partial x}=K_{x} \frac{\partial^{2} c}{\partial x^{2}}+K \frac{\partial^{2} c}{\partial y^{2}}+K K_{z} \frac{\partial^{2} c}{\partial z^{2}}
$$

subject to the boundary condition determined by the washout. If the flux at the surface caused by washout is proportional to the surface concentration given by Equation 6 , then the lower boundary condition for Equation 7 is

$$
\text { Flux }\left.\right|_{z=0}=-K_{z} \frac{\partial c}{\partial z}(x, y, z=0, t)=-V_{w} c(x, y, z=0, t)
$$

or

$$
\begin{aligned}
\frac{\partial c}{\partial z}-A c & =\left.0\right|_{z=0} \\
A & \equiv \frac{V_{W}}{K_{z}}
\end{aligned}
$$

The constant $A$ has the dimensions of length $^{-1}$ and may be made nondimensional by forming the product $\gamma_{\mathrm{w}} \equiv \sigma_{\mathrm{z}} \mathrm{A}$, hereafter called the washout number, where $\sigma_{z}$ is the standard deviation of the concentration within a free puff, given by $\sigma_{z}^{2}=2 K_{z} t$. As will be shown later, the washout number determines the concentration of HTO downwind from a low-level source.

The advection term $U \frac{\partial c}{\partial x}$ (Equation 7) may be removed by transforming Equation 7 to the ${ }^{2}$ moving coordinate system $\mathrm{X}=\mathrm{x}-\mathrm{Ut}$, yielding

$$
\frac{\partial c}{\partial t}=K_{x} \frac{\partial^{2} c}{\partial X^{2}}+K \frac{\partial^{2} c}{\partial y^{2}}+K_{z} \frac{\partial^{2} c}{\partial z^{2}}
$$

The solution for an unbounded puff is

$$
c(x, y, z, t)=\frac{(2 \pi)^{-3 / 2}}{\sigma_{x} \sigma^{\sigma}{ }^{\sigma} z} Q_{0} \exp \left[\left(-\frac{x^{2}}{2 \sigma_{x}{ }^{2}}+\frac{y^{2}}{2 \sigma_{y}{ }^{2}}+\frac{-z^{2}}{2 \sigma_{z}{ }^{2}}\right)\right]
$$


In Equation 11, $Q_{0}$ is the amount of material expelled from the source at $(0,0,0)$ at the initial time $t=0$ (Roberts), ${ }^{5}$ and $\sigma_{x}$ and $\sigma_{y}$ are defined analogously to $\sigma_{z}$.

If the source is at a height $h$, then total reflection of the flux at the surface requires placing an image source at $z=-h$ resulting in

$$
\begin{gathered}
c(X, y, z, t)=\frac{(2 \pi)^{-3 / 2}}{\sigma_{x}{ }^{\sigma} y^{\sigma} z} Q_{0}\left\{\exp \left[-\left(\frac{x^{2}}{2 \sigma_{x}^{2}}+\frac{y^{2}}{2 \sigma_{y}^{2}}\right)\right]\right. \\
\left.X \exp \left[-\frac{(z-h)^{2}}{2 \sigma_{z}^{2}}\right]+\exp \left[-\frac{(z+h)^{2}}{2 \sigma_{z}^{2}}\right]\right\}
\end{gathered}
$$

However, because washout produces a net surface flux, a line of images extending from $-\mathrm{h}$ to a depth infinitely below the surface must be added to the single image. The image source strength must be negative because the surface flux is negative. Denoting the source strength per unit length by $Q(z)$, the resulting concentration from all the sources is

$$
\begin{array}{r}
c(X, y, z, t)=\frac{(2 \pi)^{-3 / 2}}{\sigma_{x} y^{\sigma} z} Q_{0} \exp \left[-\left(\frac{X^{2}}{2 \sigma_{x}^{2}}+\frac{y^{2}}{2 \sigma_{y}^{2}}\right)\right] \\
X\left\{\exp \left[-\frac{(z-h)^{2}}{2 \sigma_{z}^{2}}\right]+\exp \left[-\frac{(z+h)^{2}}{2 \sigma_{z}^{2}}\right]-R\right\}
\end{array}
$$

$R$ is the effect of the line of images and is given by

$$
R=-\int_{-\infty}^{-h} \mathrm{~d} z^{\prime} \hat{Q}\left(z^{\prime}\right) \exp \left(-\frac{\left(z-z^{\prime}\right)^{2}}{2 \sigma z^{2}}\right)
$$

To solve for $\hat{Q}\left(z^{\prime}\right)$, a differential equation with the aid of the surface boundary condition (Equation 9) must be constructed. The factors in $x$ and $y$ are omitted to simplify the algebra. The result for the surface concentration is

$$
\begin{gathered}
c(z=0, t)=\frac{(2 \pi)^{-\frac{1}{2}}}{\sigma}\left[2 \exp \left(\frac{-h^{2}}{2 \sigma_{z}^{2}}\right)+\int_{-\infty}^{-h} d z^{\prime} \hat{Q}\left(z^{\prime}\right)\right. \\
\left.X \exp \left(-\frac{z^{\prime 2}}{2 \sigma_{z}^{2}}\right)\right] \\
-10-
\end{gathered}
$$


The surface flux term must be carefully handled because the derivative is taken with respect to $z$, and the integral is computed with respect to $z^{\prime}$. By invoking the Leibnitz rule for differentiating with respect to a parameter

$$
\begin{aligned}
& \frac{d}{d \alpha} \int_{g_{1}(\alpha)}^{g_{2}(\alpha)} d x f(x ; \alpha)=\int_{g_{1}(\alpha)}^{g_{2}(\alpha)} d x \frac{\partial f}{\partial \alpha}(x ; \alpha)+ \\
& \quad f\left(g_{2} ; \alpha\right) \frac{d g_{2}}{d \alpha}-f\left(g_{1} ; \alpha\right) \frac{d g_{1}}{d \alpha}
\end{aligned}
$$

and changing variables in Equations 13 and 14 and to $r \equiv z^{\prime}-z$, it follows that

$$
\begin{gathered}
\frac{\partial}{\partial z} \int_{-\infty}^{-h-z} \operatorname{drQ}(r+z) \exp \left(\frac{-r^{2}}{2 \sigma_{z}^{2}}\right)=\int_{-\infty}^{-h-z} d r \frac{d \hat{Q}(r+z)}{d z} \\
X \exp \left(\frac{-r^{2}}{2 \sigma_{z}^{2}}\right)-\hat{Q}(-h) \exp \left[\frac{-(h+z)^{2}}{2 \sigma_{z}^{2}}\right]
\end{gathered}
$$

At the surface, the right side of Equation 17 becomes

$$
\int_{-\infty}^{-h} d z^{\prime} \frac{d \hat{Q}}{d z^{\prime}} \exp \left(\frac{-z^{\prime 2}}{2 \sigma_{z}^{2}}\right)-\hat{Q}(-h) \exp \left(\frac{-h^{2}}{2 \sigma_{z}^{2}}\right)
$$

Invoking the surface boundary condition gives

$$
\frac{d \hat{Q}}{d z^{\prime}}\left(z^{\prime}\right)=A \hat{Q}\left(z^{\prime}\right) \text { and } \hat{Q}\left(z^{\prime}=-h\right)=-2 A
$$

Therefore, the image source distribution is

$$
\begin{aligned}
\hat{Q}\left(z^{\prime}\right)= & -2 A \exp \left[A\left(h+z^{\prime}\right)\right] ; \text { the concentration } c(z, t) \\
\text { is } c(z, t)= & \frac{(2 \pi)^{-\frac{1}{2}}}{\sigma_{z}}\left\{\exp \left[-\frac{(z-h)^{2}}{2 \sigma_{z}}\right]+\exp \left[-\frac{(z+h)^{2}}{2 \sigma_{z}^{2}}\right]\right. \\
& \left.-2 A e^{A h} \int_{-\infty}^{-h} d z^{\prime} \exp \left(A z^{\prime}\right) \exp \left[-\frac{\left(z-z^{\prime}\right)^{2}}{2 \sigma z^{2}}\right]\right\}(21)
\end{aligned}
$$


The integral on the right side of Equation 21 can be evaluated by transforming the variable of integration again to $r$. The result can be expressed in terms of a complementary error function, erfc,

$$
\begin{aligned}
R(z)= & (2 \pi)^{\frac{1}{2}} A \sigma_{z} \exp \left[\frac{A^{2} \sigma_{z}^{2}}{2}+A(h+z)\right] \\
& X \operatorname{erfc}\left[\frac{\sqrt{2}}{2}\left(A \sigma_{z}+\frac{h+z}{\sigma_{z}}\right)\right] \\
& \operatorname{erfc}(x) \equiv 2(\pi)^{-\frac{1}{2}} \int_{x}^{\infty} d t e^{-t^{2}}
\end{aligned}
$$

The surface concentration is a function of the washout number $\gamma_{\mathrm{W}}=A \sigma_{\mathrm{Z}}$ and is given by

$$
\begin{aligned}
& c(x, y, z=0, t)=\frac{Q_{0}(2 \pi)^{-3 / 2}}{\sigma_{x} \sigma_{y} \sigma_{z}} \exp -\left[\frac{(x-U t)^{2}}{2 \sigma_{x}^{2}}+\frac{y^{2}}{2 \sigma_{y}{ }^{2}}\right] \\
& X\left\{2 \exp \left(\frac{-h^{2}}{2 \sigma_{z}{ }^{2}}\right)-(2 \pi)^{\frac{1}{2}} \gamma_{w} \exp \left(\frac{\gamma_{w}{ }^{2}}{2}+A h\right)\right. \\
& \left.X \operatorname{erfc}\left[\frac{\sqrt{2}}{2}\left(\gamma_{w}+\frac{h}{\sigma_{z}}\right)\right]\right\}
\end{aligned}
$$

Fickian diffusion is not required in the horizontal plane when Equation 23 is applied to practical calculations; the boundary value condition (Equation 8 ) only requires $K_{z}$ to be constant. In Equation 23, $\sigma_{x}$ and $\sigma_{y}$ may be supplied by field data which are unlikely to be Fickian. The restriction of constant $K_{z}$ will be removed later.

\section{TOTAL SURFACE WASHOUT FROM THE FICKIAN PUFF}

The amount of material deposited at the surface on a unit area by washout is the time integral of the surface flux from $t=0$ to $t=\infty$; thus,

$$
\Psi(x, y) \equiv V_{W} \int_{t=0}^{t=\infty} d t c(x, y, z=0, t)
$$


The calculation is performed by replacing the integration over $t$ by an integration over $x$,

$$
\Psi(x, y)=\frac{(\pi)^{-\frac{1}{2}} V_{w}}{U \sigma \sigma_{x}} \int_{-\infty}^{x} d X \exp \left(-\frac{x^{2}}{2 \sigma_{x}{ }^{2}}\right) c(y, h)
$$

The contribution to the surface concentration by $y$ and $h$ is represented by $c(y, h)$ in Equation 25 . The integration is simplified by making the usual approximation that the integrand is negligible except in the vicinity of $X=0(x=U t)$. In the vicinity of $X=0$, the standard deviations $\sigma_{x}, \sigma_{y}$, and $\sigma_{z}$ are almost constant. This approximation yields

$$
\psi(x, y)=\frac{Q_{o} V_{w}}{2 \pi \sigma_{y} \sigma U} \exp \left(\frac{-y^{2}}{2 \sigma_{z}^{2}}\right)\left[2 \exp \left(\frac{-h^{2}}{2 \sigma_{z}^{2}}\right)-R\right]
$$

multiplied to a factor equal to

$$
\frac{1}{2}\left[1+\operatorname{erfc}\left(\frac{x}{\sqrt{2} \sigma_{x}}\right)\right]
$$

For $x>>\sigma_{x}$, this expression is almost unity.

Equation 26 shows that in the special case of a very small washout number (light rain), the total washout under the puff equals the washout generated per unit time by a plume if the $\sigma_{y, z}$ for a puff equals the $\sigma_{y}, z$ for a plume. For $\gamma_{w}>1$ (large washout numbers), the line of images almost collapses to a point source located at $z=-h$ with a source strength equal to $-2 \mathrm{Q}$. Thus, the surface concentration almost vanishes.

\section{THE LEAKY PUFF MODEL}

Constant $K_{z}$ was assumed for the analysis in the preceding two sections. If $\mathrm{K}_{\mathrm{z}}$ were constant, then $\sigma_{z}$ would be proportional to $x^{\frac{1}{2}}$, a relation that is not supported by field experiments.

The ad hoc approach advanced in this section is similar to that currently used for the dry deposition of plumes. ${ }^{6}$ The concentration is assumed to be Gaussian and satisfies a continuity equation. The initial source strength is replaced by an everdiminishing "effective source strength." The Leaky Puff model can give results quite different from the Fickian Puff model. However, the differences are small for light rain and nearly Fickian diffusion. 
A conservation equation is written to derive a differential equation for the effective source strength. The material lost to the ground is manifested in a reduced effective source strength $Q_{\text {eff }}(t)$. The equation is

$$
\int_{0}^{t} \int_{-\infty}^{\infty} \int_{-\infty}^{\infty} d x d y d t v_{w} c(x, y, z=0, t)=Q_{0}-Q_{e f f}(t)
$$

In Equation $28, Q_{0}$ is the amount of material emitted from the stack at $t=0 ; Q f_{f}(t)$ is the amount of material remaining

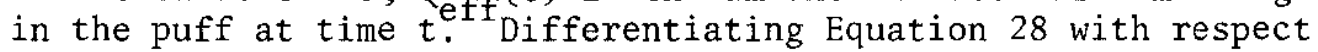
to $t$ gives

$$
V_{w} \int_{-\infty}^{\infty} \int_{-\infty}^{\infty} d x d y c(x, y, z=0, t)=-\dot{Q}_{\text {eff }} .
$$

The expression for a Gaussian Puff with a time-varying total mass is

$$
\begin{aligned}
c(x, y, z=0, t)= & \frac{2(2 \pi)^{-3 / 2}}{\sigma_{x} y^{\sigma} z} Q_{e f f}(t) \exp \left[-\left(\frac{x^{2}}{2 \sigma_{x}{ }^{2}}+\frac{y^{2}}{2 \sigma x^{2}}\right.\right. \\
& \left.\left.+\frac{h^{2}}{2 \sigma_{x}^{2}}\right)\right]
\end{aligned}
$$

Substitution into Equation 29 leads to

$$
\dot{Q}_{e f f}(t)+\left[\left(\frac{2}{\pi}\right)^{\frac{1}{2}} v_{w} \frac{\exp \left(\frac{-h^{2}}{2 \sigma_{z}^{2}}\right)}{\sigma_{z}}\right] Q_{e f f}=0
$$

The solution is

$$
Q_{e f f}(t)=Q_{0} \exp -\left[\left(\frac{2}{\pi}\right)^{\frac{1}{2}} v_{w} \int_{0}^{t} d t^{\prime} \frac{\exp \left(\frac{-h^{2}}{2 \sigma^{2}}\right)}{\sigma_{z}}\right]
$$

Changing the variable of integration yields

$$
Q_{\text {eff }}(x)=Q_{0}\left[\exp \int_{0}^{x} d x \cdot \frac{\exp \left(\frac{-h^{2}}{2 \sigma_{z}^{2}}\right)}{\sigma_{z}}\right]-\left(\frac{2}{\pi}\right)^{\frac{1}{2}}\left(\frac{V_{W}}{U}\right)
$$


a result which is identical to that given in Reference 6 for a Gaussian Puff. Although the integral involved in Equation 33 is nonelementary when $\sigma_{z}$ is given by a power law and $h>0$, for the special case of a surface release and Fickian diffusion, Equation 33 reduces to

$$
Q_{e f f}(x)=Q_{0} \exp \left[-\left(\frac{2}{\pi}\right)^{\frac{1}{2}} \frac{V_{w} x}{U \sigma_{z}}\right]
$$

The exponential-like decay with distance from the source in the Leaky Puff model is in marked contrast to the Fickian Puff model. When the rain is light $\left(\gamma_{W}\right.$ sma11), Equation 34 may be approximated by the first two terms of a Taylor series,

$$
Q_{\text {eff }} \doteq Q_{0}\left[1-\left(\frac{2}{\pi}\right)^{\frac{1}{2}} \frac{V_{w} x}{U \sigma_{z}}\right]=Q_{0}\left[1-\left(\frac{2}{\pi}\right)^{\frac{1}{2}} \gamma_{w}\right]
$$

An effective source strength can also be computed for the Fickian Puff when $h=0$. For the Fickian Puff, Equation 23 gives

$$
Q_{e f f}=Q_{0} e^{\frac{\gamma_{w}{ }^{2}}{2}} \operatorname{erfc}\left(\frac{\gamma_{w}}{\sqrt{2}}\right) \doteq Q_{0}\left[1-\left(\frac{2}{\pi}\right)^{\frac{1}{2}} \gamma_{w}\right]
$$

Although Equations 34 and 36 are the same for small $\gamma_{W}$, large $\gamma_{w}$ gives a quite different effect. Equation 34 remains an exponential, but the middle term of Equation 36 becomes

$$
\mathrm{Q}_{\mathrm{eff}}(\mathrm{x}) \sqrt[\sim]{\frac{2}{\pi}} \frac{\mathrm{Q}_{0}}{\gamma_{\mathrm{w}}}
$$

Equations 34 and 37 show that the Leaky Puff is exhausted asymptotically at a much more rapid rate than the Fickian Puff. This discrepancy, while surprising, is not large for near-Fickian diffusion and for moderate values of $\gamma_{W}$.

The relation for the total washout for a leaky Fickian Puff emitted from a ground source is

$$
\psi(x, y ; h=0) \doteq \frac{V_{w} Q_{0} \exp \left[-2\left(\frac{2}{\pi}\right)^{\frac{1}{2}} \frac{V_{w} x}{U \sigma_{z}} \exp \left(\frac{-y^{2}}{2 \sigma_{y}^{2}}\right)\right]}{\pi \sigma_{z} \sigma_{y} U}
$$


An expression can be written for the washout for arbitrary $h>0$ and $\sigma_{z}=\beta \mathrm{x}^{\mathrm{p}}$, but the result involves an exponential of an incomplete gamma function and is not particularly illuminating.

In symbolic form it is

$$
\psi(x, y ; h=0) \stackrel{\bullet}{=} \frac{V_{w} Q_{e f f}(x)}{\pi \sigma_{z} \sigma_{y} U} \exp \left\{-\left[\frac{y^{2}}{2 \sigma_{y}^{2}}+\frac{h^{2}}{2 \sigma_{z}^{2}}\right]\right\}
$$
For the case of a surface release and $\sigma_{z}=\beta x^{p}$, simple algebra
yields

$$
\Psi(x, y ; h=0) \doteq \frac{V_{w} Q_{0}}{\pi \sigma_{z} \sigma_{y} U} \exp \left\{-\left[\frac{y^{2}}{2 \sigma_{y}^{2}}+\left(\frac{2}{\pi}\right)^{\frac{1}{2}} \frac{V_{w} x}{(1-p) U \sigma_{z}}\right]\right\}
$$

For moderate downwind distances, an approximate expression for the washout from an elevated source shows that the washout reduces to Equation 39 for all reasonable stack heights. Equation 40 shows that the Leaky Puff model fails for $p>1$.

\section{THE LEAKY GARBAGE CAN MODEL}

The concentration in the Leaky Garbage Can model is reduced in a manner similar to the Leaky Puff mode1. However, the material is assumed to be well mixed throughout the mixed layer with a rigid lid at $z=H$. The conservation equation is as in Equation 28

$$
\int_{0}^{t} \int_{-\infty}^{\infty} \int_{-\infty}^{\infty} c d x d y d t=Q_{0}-Q_{e f f},
$$

but the concentration is given now by

$$
\begin{array}{ll}
c(X, y, z, t)=\frac{(2 \pi)^{-1}}{\sigma_{x} \sigma_{y} H} Q_{\text {eff }} \exp \left\{-\left(\frac{x^{2}}{2 \sigma_{x}{ }^{2}}+\frac{y^{2}}{2 \sigma_{y}{ }^{2}}\right)\right\}, & z \leq H \\
c(x, y, z, t)=0 & , z>H
\end{array}
$$

The differential equation for $Q_{\text {eff }}$ simplifies to

$$
\dot{Q}_{\text {eff }}+\frac{V_{w}}{H} Q_{e f f}=0
$$


Thus,

$$
\begin{aligned}
\psi(x, y)=V_{w} \int_{0}^{\infty} d t c(x, y, z=0, t) \doteq & \frac{(2 \pi)^{-\frac{1}{2}} Q_{0} V_{w}}{H \sigma_{y} U} \\
& {\left[\exp \left(\frac{-y^{2}}{2 \sigma_{y}{ }^{2}}-\frac{V_{w} x}{U H}\right)\right] }
\end{aligned}
$$

The Leaky Garbage Can model shows simple exponential decay and can be evaluated in terms of elementary functions. The washout number for the Leaky Gargage can model is

$$
\gamma_{w}=\frac{V_{w} x}{U H} \text {, }
$$

suggesting a scale length $L_{W}=\frac{U H}{V}$ over which the puff is washed out to a factor of $1 / \mathrm{e}$ of its original strength. This scale length was derived by simple dimensional reasoning.

\section{APPROXIMATIONS AND NUMERICAL CALCULATIONS}

Results from the three models for selected rainfall rates, advection velocities, and stack heights are presented. An approximate relation for the effect of an elevated source in the Leaky Puff model is obtained, and the extension of the Fickian Puff model to diffusion other than Fickian is justified. In addition, the Leaky Garbage Can model will be generalized for a rising lid.

\section{Fickian Puff Model}

Routine evaluation of the Fickian Puff relation (Equation 26) leads to computer overflow and underflow. The overflow and underflow are caused by the exponential in the defining relation for $R$ (Equation 22) becoming too large and the complementary error function becoming too small for the computer to handle. Their product, however, remains within range of the computer. An asymptotic form removes the difficulty.

When the argument of the complementary error function is large, it may be approximated by the first term of its asymptotic expansion

$$
\operatorname{erfc}(t) \sim\left(\pi t^{2}\right)^{-\frac{1}{2}} \exp \left(-t^{2}\right)
$$


Equation 46 used in Equation 47 and the limiting inequality $\gamma_{\mathrm{w}} \gg \frac{\mathrm{h}}{\sigma_{z}}$ lead to

$$
R \sim \frac{2}{\left(1+\frac{h U}{2 V_{W} x}\right)} \exp \left(\frac{-h^{2}}{2 \sigma_{z}^{2}}\right)
$$

Relation 47 simplifies the evaluation of Equation 26 far downstream from the source; Equation 26 reduces to

$$
\psi(x, y) \sim \frac{Q_{0} V_{w}}{\pi \sigma_{y} \sigma_{z} U} \exp \left[-\left(\frac{y^{2}}{2 \sigma_{y}^{2}}+\frac{h^{2}}{2 \sigma_{z}^{2}}\right)\right]\left[\frac{q}{1+q}\right]
$$
in which $\mathrm{q} \equiv \mathrm{hU} /\left(2 \mathrm{~V}_{\mathrm{w}} \mathrm{x}\right)$. Equation 48 eliminates overflow and
underflow.

$\mathrm{K}_{\mathrm{x}}$, $\mathrm{K}_{\mathrm{y}}$, and $\mathrm{K}_{z}$ were assumed to be constant in the derivation leading to the Fickian Puff model. Because $\sigma_{\mathrm{x}}$ and $\sigma_{\mathrm{y}}$ do not enter directly into the boundary value problem, this requirement may be discarded partially as unnecessary. $\sigma_{x}$ and $\sigma_{y}$ may be supplied by field measurements like Högström's where the G's are the power law form

$$
\sigma_{i}=\beta_{i} x^{p_{i}} \text {. }
$$

The requirement that $K_{z}$ be constant may also be dropped after a simple heuristic argument ${ }^{z}$ is invoked. The coordinate system $(x, y, z, t)$ moves with the puff in which $x=x-U t$. The expression for the vertical diffusion of the puff in this system is

$$
\frac{\partial}{\partial z}\left[K \frac{\partial c}{\partial z}(z, t)\right]=\frac{\partial c}{\partial t}(z, t)
$$

If $K_{z}$ is given by a power law in time, $k_{z}=\alpha t^{m}$,

and a change is made to a hypothetical time variable $\hat{t}$ given by

$$
\hat{t}=t^{r}
$$

then Equation 49 becomes

$$
\alpha \hat{t} \frac{m}{r} \frac{\partial^{2} c}{\partial z^{2}}=r \hat{t}^{(1-1 / r)} \frac{\partial c}{\partial \widehat{t}}
$$


Because $r$ is arbitrary, it may be chosen to remove the dependence of Equation 52 on $\hat{t}$. This gives $r=m+1$, and

$$
\frac{\alpha}{r} \frac{\partial^{2} c}{\partial z^{2}}=\frac{\partial c}{\partial t}
$$

The diffusion coefficient $\hat{K}_{z}$ in the time-transformed system is constant and is

$$
\hat{K}_{z}=\frac{\alpha}{r}=\frac{\alpha}{m+1}
$$

Equations 53 and 54 show that the diffusion is Fickian provided $\hat{t}=t^{m+1}$.

The time power law (Equation 51 ) in the moving coordinate frame is related to the spatial power law, determined empirically,

$$
\sigma_{z}=\beta \mathrm{x}^{\mathrm{P}}
$$

by the relation

$$
\sigma_{z}(t)=\beta U^{P} t^{P}
$$

Because $\sigma_{z}(t)$ is a geometrical quantity, it must be equal to $\hat{\sigma}_{z}(\hat{t})$ at the same physical time. Thus,

$$
\hat{\sigma}(\hat{t})=\sigma(t)=\left(2 \hat{K}_{z} \hat{t}\right)^{\frac{1}{2}}
$$

yields

$$
r=2 \mathrm{P} \text { and } \alpha=P B^{2} \mathrm{U}^{2 \mathrm{P}}
$$

The boundary condition (Equation 9),

$$
\frac{\partial c}{\partial z}(z=0, t)=\frac{V_{W}}{K_{z}(t)} c(z=0, t)
$$

must be transformed to the new time system by replacing $K_{z}(t)=a t^{m}$ with its equivalent form $K_{z}=\hat{k}_{z} r t^{r-1}$. The rain rate which is constant in the $t$ system is variable in the $t$ system and is given by

$$
V_{w} \equiv \frac{d W}{d t}=\frac{d W}{d \hat{t}} \frac{d \hat{t}}{d t}=\hat{v}_{w} r t^{r-1}
$$


In Equation $60, W$ is the total amount of rainfall for a unit area. A cancellation in Equation 59 leads to

$$
\frac{\partial c}{\partial z}(z=0, \hat{t})=\frac{\hat{V}_{w}}{\hat{K}_{z}} c(z=0, \hat{t})=\hat{A} c(z=0, \hat{t})
$$

Thus,

$$
\begin{aligned}
& \hat{R}=(2 \pi)^{\frac{1}{2}} \hat{A} \sigma_{z} \exp \left(\frac{\hat{A}^{2} \sigma_{z}{ }^{2}}{2}+\hat{A} h\right) \operatorname{erfc}\left[\frac{\sqrt{2}}{2}\left(\hat{A} \sigma_{z}+\frac{h}{\sigma_{z}}\right)\right] \\
& A=\frac{V W}{P \sigma_{z}{ }^{2}}
\end{aligned}
$$

The washout relations for a Fickian Puff are approximately valid for arbitrary $\sigma_{x}, \sigma_{y}$, and a power 1 aw $\sigma_{z}$.

\section{Leaky Puff Model}

The derivation leading to the expression for the effective source strength (Equation 33) is very simple. However, the exact evaluation of the integral in Equation 33 for an arbitrary power $1 \mathrm{aw} \sigma_{7}$ and for $\mathrm{h}>0$ requires special functions. A simple asymptotic expression for the effective source strength will be derived valid for $\sigma_{z}>>h$.

If $\sigma_{z}$ is given by Equation 55 , then the integral which must be approximated is

$$
I(X)=\int_{0}^{X} d x x^{-P} \exp \left(-\alpha x^{-2 P}\right)
$$

In Equation 63, $\alpha \equiv \frac{\mathrm{h}^{2}}{2} \beta^{-2}$. When Equation 63 is integrated by parts, the result is

$$
I=\frac{1}{1-P} x^{1-P} \exp \left(-\alpha X^{-2 P}\right)-\frac{2 \alpha P}{1-P} \int_{0}^{X} d x x^{-3 P} \exp \left(-\alpha x^{-2 P}\right)
$$


The first term on the right side of Equation 64 also results from holding the exponential factor constant in Equation 63 . Equation 64 may be motivated by noting that the exponential factor is nearly unity and relatively constant for large $X$. The integration by parts can be continued. The result is

$$
\begin{aligned}
& I=T_{i}+T_{2}+T_{3}+I_{4} \text { in which } \\
& T_{1}(x)=\frac{1}{1-P} x^{1-P} \exp \left(-\alpha x^{-2 P}\right), \\
& T_{2}(x)=\frac{2 \alpha P}{(1-P)(1-3 P)} x^{1-3 P} \exp \left(-\alpha x^{-2 P}\right), \\
& \mathrm{T}_{3}(\mathrm{x})=\frac{(2 \alpha P)^{2}}{(1-\mathrm{P})(1-3 \mathrm{P})(1-5 \mathrm{P})} \mathrm{x}^{1-5 \mathrm{P}} \exp \left(-\alpha \mathrm{x}^{-2 \mathrm{P}}\right) \text {, and } \\
& I_{4}(x)=-\frac{(2 \alpha P)^{3}}{(1-P)(1-3 P)(1-5 P)} \int_{0}^{X} d x^{\prime} x^{, 1-7 P} \exp \left(-\alpha x^{,-2 P}\right) .
\end{aligned}
$$

The terms $T_{1}, T_{2}$, and $T_{3}$ are the first three terms in an asymptotic expression for $I(X)$. Each of the terms is finite for a $11 \frac{1}{3}<\mathrm{p}<1$.

The ratio of two consecutive terms shows that the terms diminish in magnitude for large $X$. For example, the ratio

$$
\left|\frac{\mathrm{T}_{2}}{\mathrm{~T}_{1}}\right|=\frac{2 \alpha \mathrm{P}}{3 \mathrm{P}-1}\left(\frac{1}{\mathrm{x}^{2 \mathrm{P}}}\right)
$$

shows that as $x$ increases, the second term becomes smaller compared to the first term. Approximating the integral by the first two terms in the asymptotic expression leads to a value of $Q_{\text {eff }}(x)$ given by

$$
\begin{aligned}
& Q_{\text {eff }}(x) \sim Q_{0} \exp \left\{-\left(\frac{2}{\pi}\right)^{\frac{1}{2}} \frac{V_{W}}{U}\left[\frac{x}{(1-P) \sigma_{z}} \exp \left(-\frac{h^{2}}{2 \sigma_{z}^{2}}\right)\right.\right. \\
& \left.\left.X\left[1+\frac{P}{(3 P-1)}\left(\frac{h}{\sigma_{z}}\right)^{2}+\ldots\right]-\left(\frac{(h / \sqrt{2})^{\frac{1-P}{P}}}{\frac{1}{P}}\right) \Gamma\left(\frac{3 P-1}{2 P}\right)\right]\right\}
\end{aligned}
$$


For a surface release, Equation 67 reduces to the exact expression given in Equation 39. Elevating the source serves to displace the position of the maximum total washout downstream from the stack.

\section{The Leaky Garbage Can Model}

Although the total washout expression for the Leaky Garbage Can model (Equation 44) was derived for a fixed mixed depth, a fixed lid is a poor assumption after a frontal passage when the mixed depth may rise rapidly.

The problem is easily solved if the mixed depth $H(t)$ is assumed to rise linearly from an initial height $H_{0}$ at $t=0$ to a maximum height $H_{m}$ at time $t_{m}$. The differential equation for $Q_{\text {eff }}(t)$ then becomes

$$
\frac{\mathrm{dQ}_{\text {eff }}}{\mathrm{Q}_{\text {eff }}}=\frac{-\mathrm{V}_{w}}{\left(\mathrm{H}_{0}+t \mathrm{H}\right)} \mathrm{dt}
$$

in which

$$
\begin{aligned}
& \dot{\mathrm{H}}=\frac{\mathrm{dH}}{\mathrm{dt}}=\frac{\mathrm{H}_{\mathrm{m}}-\mathrm{H}_{0}}{\mathrm{t}_{\mathrm{m}}} 0<\mathrm{t}<\mathrm{t}_{\mathrm{m}} \\
& \dot{\mathrm{H}}=0 \quad \mathrm{t}>\mathrm{t}_{\mathrm{m}}
\end{aligned}
$$

The solutions for Equations 68 and 69 are

$$
\begin{aligned}
& Q_{\text {eff }}(t)=Q_{0}\left(\frac{H_{0}}{H(t)}\right)^{A} \quad 0<t<t_{m} \\
& Q_{e f f}(t)=Q_{0}\left(\frac{H_{0}}{H_{m}}\right)^{A} \exp \left(-\frac{V_{w}}{H_{m}}\left(t-t_{m}\right) t_{m}>0\right)
\end{aligned}
$$

For Equation $71, \dot{H}$ is equal to its value for $0<t<t_{m}$. In Equations 70 and $71, A \equiv V_{w} / \dot{H}$. 


\section{Calculations}

Fig. 1 shows the fraction of material remaining in the Leaky Puff as a function of the downwind distance from the source. The material in the puff is rapidly depleted over the first kilometer for a surface release. Elevating the release point can greatly reduce the washout close to the source because the surface puff concentration of HTO and thus the flux are greatly reduced.

A useful property of the Leaky Puff model is the ease by which $Q_{\text {eff }}$ can be determined for a general $V_{W}$ and $U$ if a calculation has been made with a particular $V_{W}{ }^{1}, U^{1}$. From Equation 33, the general result follows from taking Qeff to the appropriate power $\mathrm{S}$

$$
\left.\begin{array}{l}
Q_{\text {eff }} / Q_{0}=\left(Q_{e f f} / Q_{0}\right)^{S} \\
S=\frac{V_{W}{ }^{1} U}{V_{W} U^{1}}
\end{array}\right\}
$$

For example, Fig. 1 shows that half the puff released from a height of $70 \mathrm{~m}$ is washed out by about $4 \mathrm{~km}$ during a $1 \mathrm{~mm} / \mathrm{hr}$ rain. Tripling the rain rate would leave $(1 / 2)^{3}=1 / 8$ of the material in the puff. Reducing the wind speed from $5 \mathrm{~m} / \mathrm{sec}$ to $1 \mathrm{~m} / \mathrm{sec}$ would leave on $1 \mathrm{y}(1 / 2)^{5}=1 / 32$ of the materia1. However, the amount of HTO remaining in the Fickian Puff is expressed by

$$
Q_{e f f}(x)=Q_{0}(1-P)
$$

$\mathrm{P}$ is a complicated function, which requires a power-series expansion in $h / \sigma_{7}$. A simple power law relationship similar to Equation 72 does not hold for Equation 73 .

Fig. 2 shows $\psi(x) / Q_{0}$, the normalized total washout for a Fickian Puff, for rain rates of $1 \mathrm{~mm} / \mathrm{hr}$ and $5 \mathrm{~mm} / \mathrm{hr}$. The washout for the heavier rain is initially greater than that of the lighter rain. Eventually (at about $40 \mathrm{~km}$ ) the heavier rain depletes the puff sufficiently that the washout from the lighter rain exceeds that from the heavier rain. The maximum washout occurs at about $3 \mathrm{~km}$ from the stack in both cases. 
The distance downwind from a stack where the maximum washout occurs $\left(x_{\max }\right)$ can be estimated provided that $Q_{\text {eff }}\left(x_{\max }\right) \approx Q_{0}\left(x_{\max }\right)$ and provided $\sigma_{y, z}$ can be expressed as power laws

$$
\begin{aligned}
& \sigma_{y}=\beta_{y} x^{P} y \\
& \sigma_{z}=\beta_{z} x^{P}
\end{aligned}
$$

The result is

$$
\begin{aligned}
& x_{\text {max }}=A h^{\frac{1}{P}} z \\
& A=2 \beta_{z}{ }^{2}\left(1+P_{y} / P_{z}\right)^{-\frac{1}{2 P_{z}}}
\end{aligned}
$$

For Fickian diffusion, doubling a stack height will quadruple the downwind distance at which the maximum washout occurs, if equilibrium theory is valid at the distance given by Equation 75 .

The washout computed from the Fickian and Leaky Puff models is compared (Fig. 3). The correspondence is surprising considering the substantial differences between the formulation of the two models. Increasing the washout number reduces the correspondence.

Fig. 4 shows the amount of HTO remaining in a puff predicted by the Leaky Garbage Can mode1. Curves are shown for a fixed mixed depth of $800 \mathrm{~m}$ and also for a mixed depth which rises in four hours from $200 \mathrm{~m}$ to $800 \mathrm{~m}$. The mixed depth is held constant after reaching $800 \mathrm{~m}$.

The washout $/ \mathrm{m}^{2}$ computed from the Fickian Puff and Leaky Garbage Can models is compared (Fig. 5). The two models did not agree for distances close to the stack because the height of the release can not be taken into account for the Leaky Garbage Can model. The HTO is assumed to be well mixed below $\mathrm{z}=\mathrm{H}$ in all cases. For distances greater than $x_{\max }$, the two correspond reasonably we11, considering the simplicity of the Leaky Garbage Can model. 


\section{CONCLUSIONS}

Three methods for estimating the equilibrium washout from an elevated instantaneous point source of tritium and tritiated water vapor were discussed. A steady wind, steady rain, and washout flux proportional to the concentration of the puff at ground level were assumed for all three models.

The Fickian Puff model initially required diffusion to be Fickian in all directions. These restrictions were abandoned first in the horizontal plane and finally in the vertical direction because of a heuristic argument. The Fickian Puff model is an approximate solution to the washout boundary value problem.

The Leaky Puff mode1 ( 1 ) forces the puff to retain its Gaussian nature regardless of how much pollutant is deposited and (2) accounts for the washed-out pollution by decreasing the source strength of the puff. The model does not satisfy the boundary values of the problem and consequently gives different results from the Fickian Puff model. The Leaky Puff model is attractive because solutions can be approximated without recourse to special functions and because the pollution can be calculated from any rain rate and wind speed $1 f$ it is known for a particular stack height, rain rate, and wind speed. The amount of pollution left, within the Leaky Puff is directly calculated by this model; whereas the Fickian Puff model would require a separate calculation with special functions.

The Leaky Garbage Can model permits Gaussian diffusion in the horizontal plane but requires the HTO to be well mixed in the vertical direction below an impenetrable lid. The concentration is assumed to be constant with height below a stable layer. These assumptions yield a fairly simple mathematical expression for the pollution remaining in the puff. The remaining pollution exhibits simple exponential decay. The half-life of the pollution in the cloud is proportional to $\mathrm{H} / \mathrm{V}_{\mathrm{W}}$.

Although results from the three models differ in detail, each shows that an HTO puff can be almost washed out within several dozen kilometers in a steady, moderate rain. Elevating the source can profoundly reduce the washout within the first several kilometers of the release. This effect is rapidly reduced at greater distances, however, and ultimately, the amount of HTO remaining in the puff is unrelated to the source height. 
The relations for the spread of the puff $\sigma_{i}(x)$ are taken from Högström's data and are chosen for their mathematical simplicity as much as for their accuracy. Although relations for distances greater than several kilometers can not be justified easily, they are used in the calculations for distances up to about $100 \mathrm{~km}$. Approximate relations for the HTO remaining in a puff can be calculated for other simple functional forms of $\sigma_{z}(x)$, but they have not been investigated.

The washout for HT and $\mathrm{T}_{2}$ can be calculated by using washout deposition velocities for tritium gas. For calculating the washout for HT and $T_{2}$, Henry's Law must be used instead of Raoult's Law. Henry's Law for hydrogen reduces the washout deposition velocity for $\mathrm{HT}$ and $\mathrm{T}_{2}$ by about six orders of magnitude below that of HTO. The fluxes from HT and $T_{2}$ are therefore small in comparison to HTO, provided Henry's Law for hydrogen is valid. The approximate equivalence of hydrogen and tritium gas is assumed and requires experimental verification. 


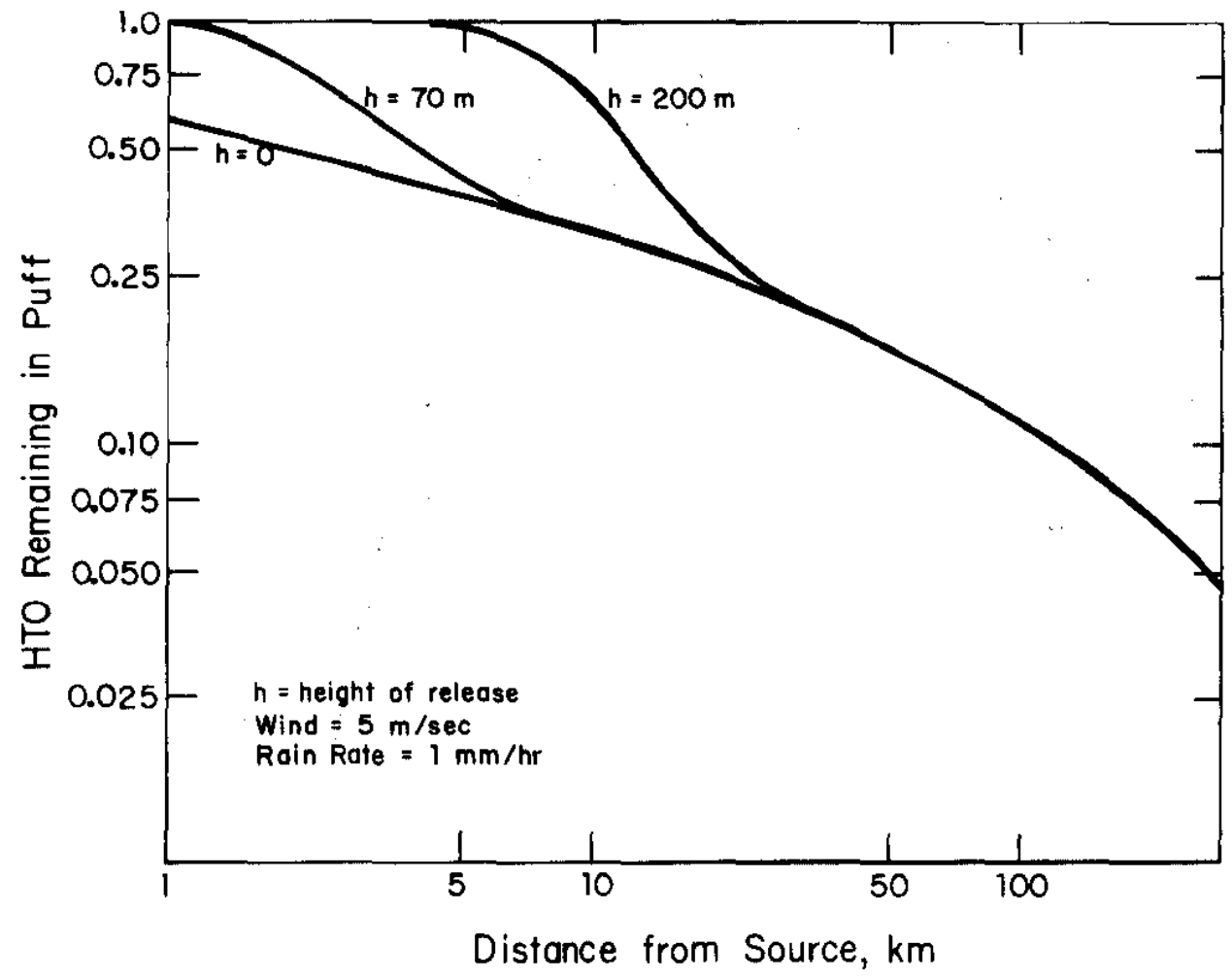

FIGURE 1. HTO Remaining in Puff Calculated by Leaky Puff Mode 


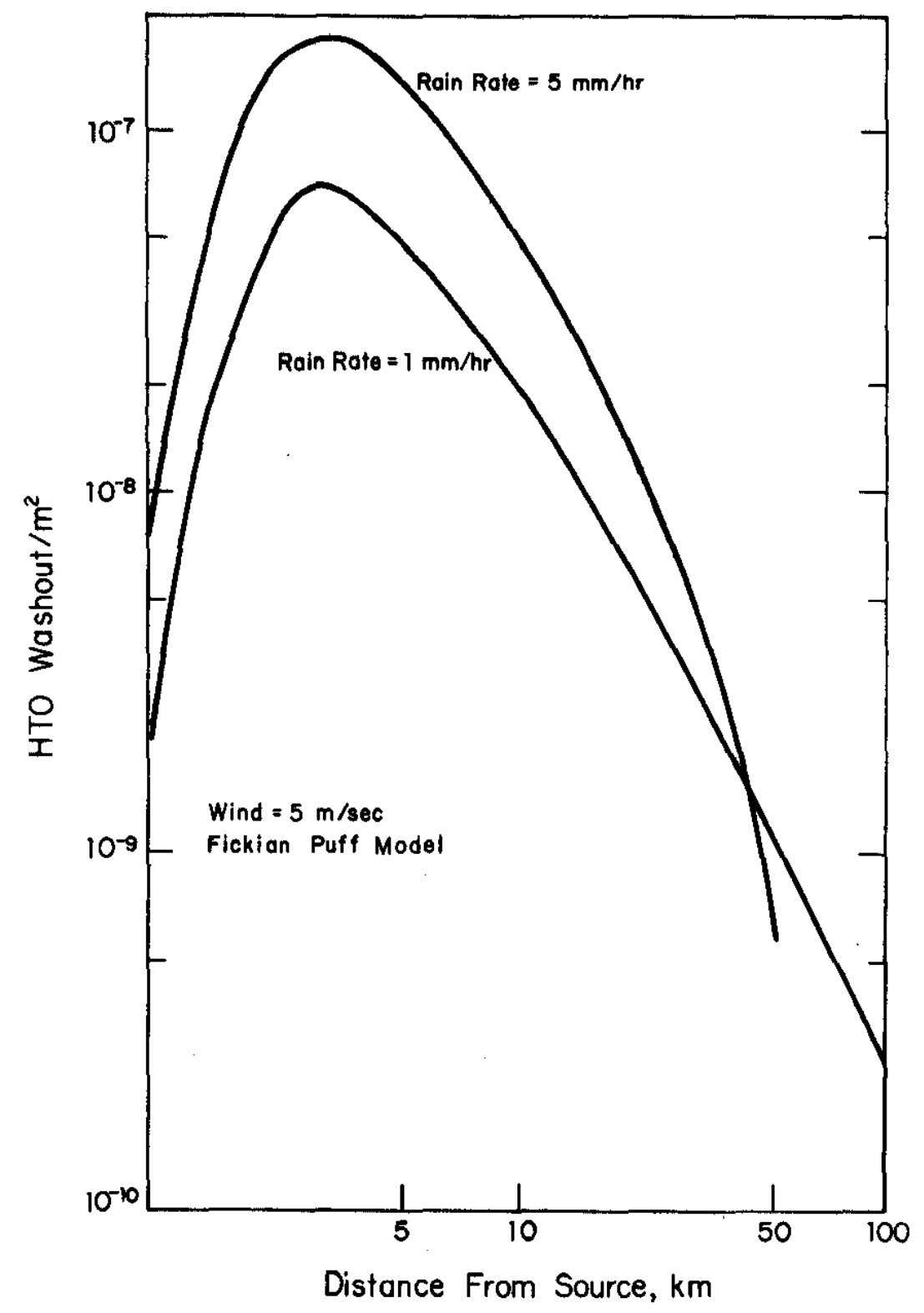

FIGURE 2. Effect of Increased Rain Rate 


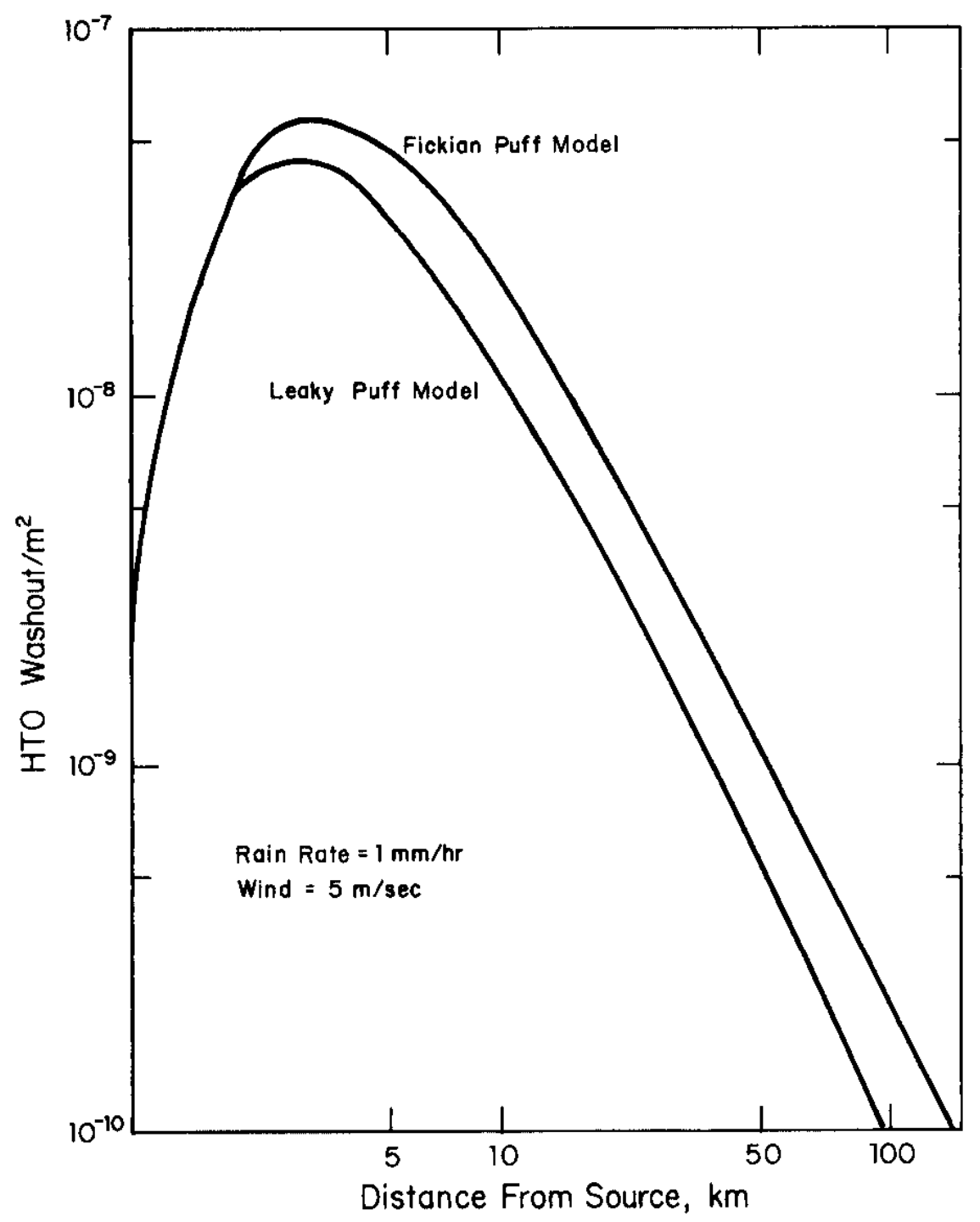

FIGURE 3. Comparison of Calculations with Fickian Puff and Leaky Puff Models 


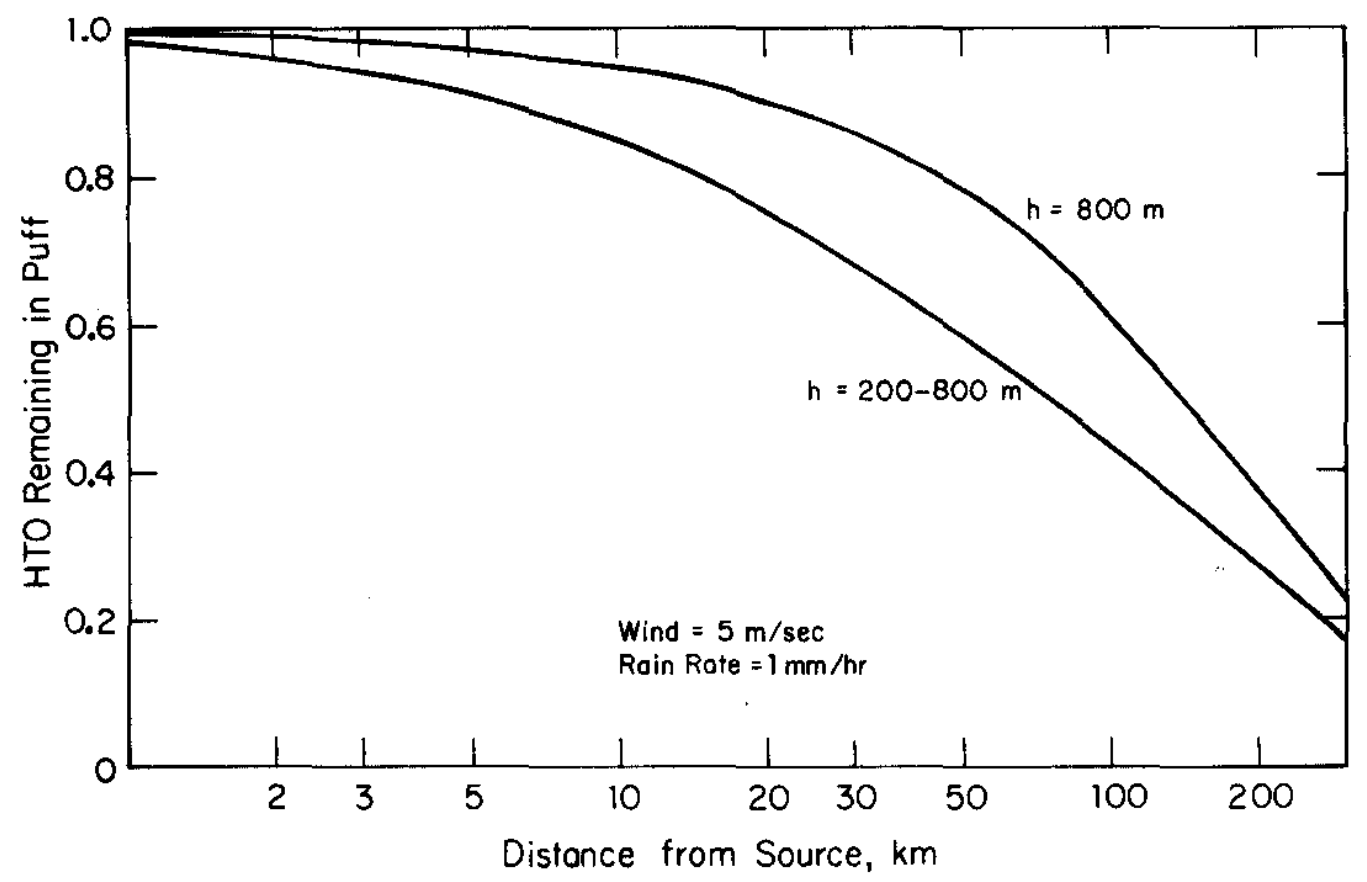

FIGURE 4. HTO Remaining in Puff Calculated by the Leaky Garbage Can Model with Fixed and Rising Lids 


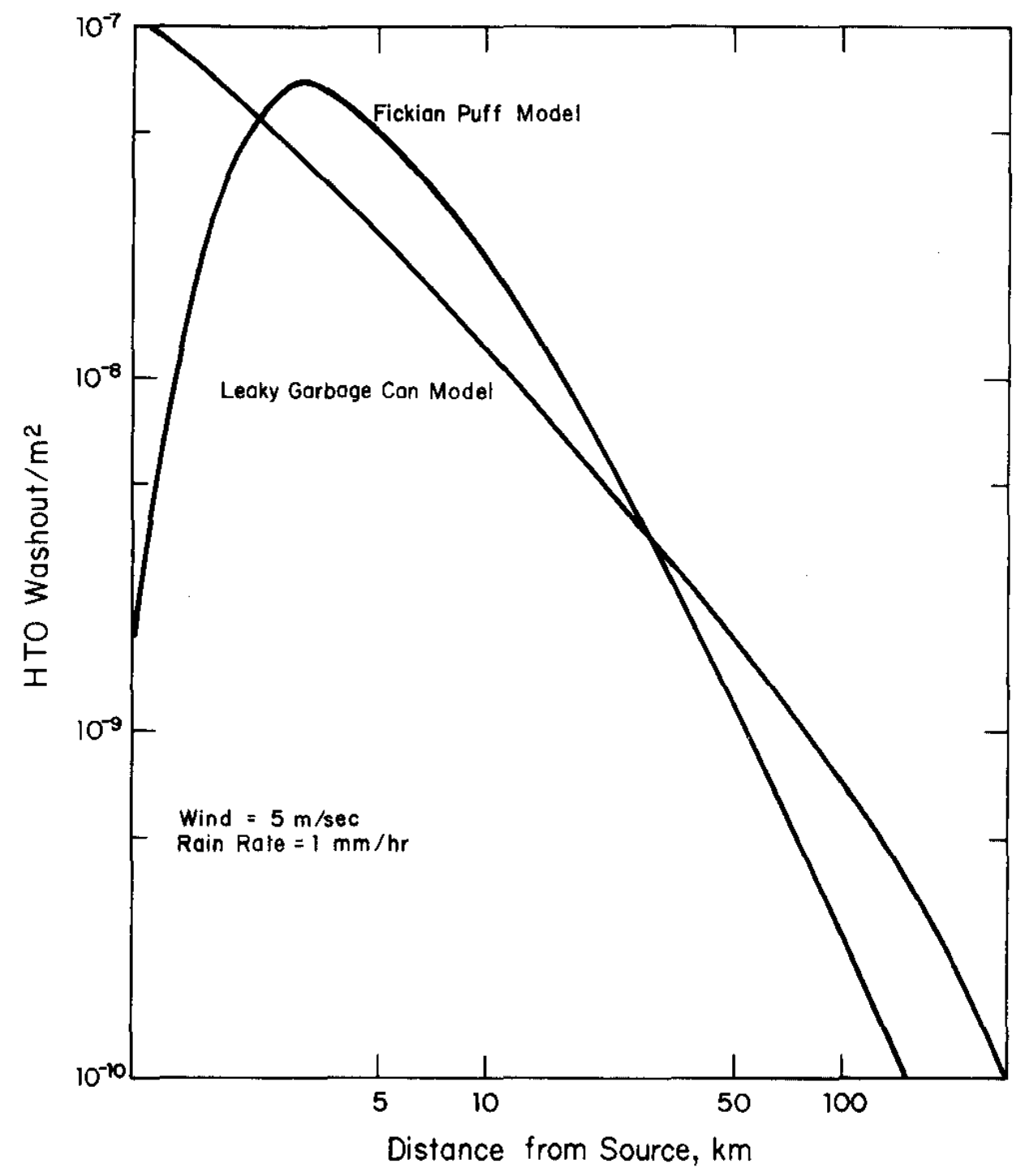

FIGURE 5. Comparison of Calculations with Fickian Puff and Leaky Garbage Can Models 


\section{REFERENCES}

1. R. J. Englemann and W. G. N. Slinn. Precipitation Scavenging (1970). CONF-700601, Nationa1 Technical Information Service, Springfield, VA (1970).

2. S. Barr. "Mesoscale Variability of Precipitation." Unpublished.

3. U. Högström. "An Experimental Study on Atmospheric Diffusion." Telzus 16(2), 205-251 (1964).

4. J. M. Hales. Scavenging of Gaseous Tritium Compounds by Rain. USAEC Report BNWL-1659, Battelle Pacific Northwest Laboratories, Richl and, WA (1972).

5. 0. F. T. Roberts. "The Theoretical Scattering of Smoke in a Turbulent Atmosphere." Proc. Roy. Soc., Ser. A, 104, 640-654 (1923).

6. D. H. Slade. Meteorology and Atomic Energy 1968. U.S. Atomic Energy Commission Office of Information Services, Oak Ridge, TN (1968). 